Pascal Dengler, Naika Foroutan

\title{
Die Aufarbeitung des NSU als deutscher Stephen-Lawrence-Moment? - Thematisierung von institutionellem Rassismus in Deutschland und Großbritannien
}

2017 | Beitrag in einem Sammelband | akzeptiertes Manuskript (Postprint) verfügbar unter https://doi.org/10.18452/22683

Finale Version folgendermaßen veröffentlicht:

Pascal Dengler, Naika Foroutan: „Die Aufarbeitung des NSU als deutscher StephenLawrence-Moment? - Thematisierung von institutionellem Rassismus in Deutschland und Großbritannien". In: Rassismuskritik und Widerstandsformen. Hrsg. von Karim Fereidooni und Meral El. Wiesbaden: Springer VS, 2017, Seiten 429-446. DOI: 10.1007/978-3-658-14721-1_25

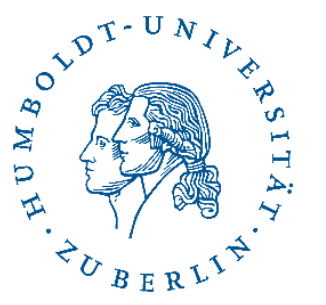

edoc-Server

Open-Access-Publikationsserver

der Humboldt-Universität zu Berlin 


\section{Die Aufarbeitung des NSU als deutscher Stephen-Lawrence- Moment? - Thematisierung von institutionellem Rassismus in Deutschland und Großbritannien}

Pascal Dengler

Naika Foroutan

\section{Einleitung}

Der rechtsterroristischen Gruppierung Nationalsozialistischer Untergrund (NSU), die nach bisherigen Erkenntnissen aus der rechtsextremen Szene der 1990er Jahre hervorging und der Uwe Mundlos, Uwe Böhnhardt und Beate Zschäpe angehörten, werden zehn Morde, die in den Städten Nürnberg, Hamburg, München, Rostock, Dortmund, Kassel und Heilbronn begangen wurden, zugerechnet. ${ }^{1}$ Neun der Opfer wurden mutmaßlich aufgrund eines rassistischen Tatmotivs ermordet; acht Personen waren türkischer, eine Person griechischer Herkunft. Darüber hinaus werden dem NSU mindestens zwei Bombenanschläge, bei denen mehrere Personen zum Teil lebensgefährlich verletzt wurden, sowie mindestens 15 bewaffnete Raubüberfälle in Sachsen, Mecklenburg-Vorpommern und Thüringen zugerechnet.

Bis zur Selbstenttarnung des NSU am 4. November 2011 war es den bundesdeutschen Ermittlungs- und Sicherheitsbehörden nicht gelungen, die Morde und Straftaten, die dem rechtsterroristischen Netzwerk inzwischen zugerechnet werden, aufzuklären. Seitdem wurden zur Aufarbeitung des NSUKomplexes sowie zur Fehleranalyse der zuständigen Ermittlungs- und Sicherheitsbehörden acht parlamentarische Untersuchungsausschüsse auf Bundes- und Länderebene eingerichtet. Davon legten vier - darunter die Untersuchungsausschüsse des Bundestags und der Landtage Bayern, Sachsen

${ }^{1}$ (1) Enver Şimşek, getötet am 9. September 2000 in Nürnberg, (2) Abdurrahim Özüdoğru, getötet am 13. Juni 2001 in Nürnberg, (3) Süleyman Taşköprü, getötet am 27. Juni 2001 in Hamburg, (4) Habil Kılıç, getötet am 29. August 2001 in München, (5) Mehmet Turgut, getötet am 25. Februar 2004 in Rostock, (6) Ismail Yaşar, getötet am 9. Juni 2005 in Nürnberg, (7) Theodoros Boulgarides, getötet am 15. Juni 2005 in München, (8) Mehmet Kubaşık, getötet am 4. April 2006 in Dortmund, (9) Halit Yozgat, getötet am 6. April 2006 in Kassel, (10) Michèle Kiesewetter, getötet am 25. April 2007 in Heilbronn. 
und Thüringen - bereits ihre Abschlussberichte vor. Neben der Rolle des Bundesamts und diverser Landesämter für Verfassungsschutz sowie der defizitären länderübergreifenden Zusammenarbeit von Ermittlungsbehörden stand vor allem der Ausschluss eines rassistischen Tatmotivs im Fokus der Kritik. Es entwickelte sich eine Kontroverse in der Frage, ob das Verhalten der Ermittlungsbehörden selbst als rassistisch bezeichnet werden könne (Vgl. Kleffner/Feser 2013). Während der Bundesvorsitzende der Deutschen Polizeigewerkschaft, Rainer Wendt, diesen Vorwurf stark kritisierte (Vgl. Bax 2013), wiesen Nebenklagevertreter_innen ${ }^{2}$ im NSU-Prozess am Oberlandesgericht München darauf hin, dass die Hinterbliebenen und Verletzten „die Anerkennung auch in der Politik, dass das systematische Versagen der Ermittlungsbehörden auf institutionellem Rassismus beruht" (von der Behrens et al. 2013), fordern, um das Problem der verschleppten Aufklärung klar als systemisches Versagen und nicht als persönliches zu benennen.

Auch wird im Zuge der Analyse und Aufarbeitung des NSU-Komplexes von verschiedenen Seiten immer wieder auf den Fall Stephen Lawrence, der in den 1990er-Jahren in Großbritannien in der öffentlichen Aufmerksamkeit stand, verwiesen. Als der rassistisch motivierte Mord an dem Schwarzen ${ }^{3}$ Jugendlichen Stephen Lawrence in London im Jahr 1993 nicht als solcher geahndet wurde und die Ermittlungen zu keiner Aufklärung führten, wurde der Fall nach dem erbrachten Beweis von Ermittlungsfehlern neu aufgerollt. Es wurde vom britischen Innenministerium eine öffentliche Untersuchungskommission unter der Leitung des ehemaligen Richters des Obersten Gerichtshofs, Sir William Macpherson of Cluny, eingerichtet, welche im Jahr 1999 ihren Abschlussbericht vorlegte - den sogenannten Macpherson Report. In ihm wurde erstmalig institutioneller Rassismus in der Metropolitian Police in London im Speziellen und in den Ermittlungsbehörden in Großbritannien im Allgemeinen identifiziert und thematisiert. Die im Bericht enthaltenen 70 Empfehlungen führten in

\footnotetext{
${ }^{2}$ Namentlich: Antonia von der Behrens, Dr. Mehmet Daimagüler, Dr. Björn Elberling, Berthold Fresenius, Alexander Hoffmann, Carsten Ilius, Detlef Kolloge, Stephan Kuhn, Angelika Lex, Stephan Lucas, Ogün Parlayan, Jens Rabe, Eberhard Reinecke, Aziz Sariyar, Sebastian Scharmer, Reinhard Schön und Peer Stolle.

${ }^{3} \mathrm{Im}$ vorliegenden Beitrag wird Schwarz großgeschrieben und weiß kleingeschrieben und kursiv gesetzt, um darauf hinzuweisen, dass sie keine biologischen Eigenschaften, sondern historisch gewachsene und sozial konstruierte Gesellschaftspositionen markieren, die mit Privilegierung oder Deprivilegierung einhergehen. Schwarz wird auch in der Verwendung als Adjektiv großgeschrieben, um das Widerstandspotenzial, das von Schwarzen Menschen und People of Color in diese Kategorie eingeschrieben wurde, sichtbar zu machen (Vgl. Eggers et al. 2009).
} 
Großbritannien zur umfangreichsten Reform im Hinblick auf das Verhältnis zwischen der Polizei und rassifizierten Personen ${ }^{4}$ (Vgl. Bowling/Phillips 2002).

In diesem Aufsatz wird der Frage nachgegangen, ob das Thema institutioneller Rassismus durch die parlamentarische Aufarbeitung des NSU-Komplexes einen ähnlich hohen Stellenwert erfuhr, wie es in Großbritannien im Rahmen des Macpherson Reports der Fall war. Dort wurden im Nachgang an die Fallaufarbeitung Gesetze auf den Weg gebracht, Maßnahmen eingeleitet und Rassismus, der sich auf einer institutionellen Ebene verorten lässt, in der Mitte der Gesellschaft diskutiert. Der Macpherson Report und der Abschlussbericht des NSU-Untersuchungsausschusses im Bundestag bilden für diese Analyse den Ausgangspunkt. ${ }^{5}$ Abschließend soll der Frage nachgegangen werden, welche Erkenntnisse sich aus dem Fall Stephen Lawrence für die Aufarbeitung und den Umgang mit dem NSU-Komplex ableiten lassen.

\section{Institutioneller Rassismus: Eine Begriffsbestimmung}

Rassistische Denk- und Handlungsweisen sind nicht Sache einzelner Individuen, sondern sind ,in der Organisation des gesellschaftlichen Zusammenlebens verortet" (Bojadžijev 2013, S. 148). Rassismus ist somit keine persönliche oder politische Einstellung, sondern muss als ein Apparat (Vgl. Terkessidis 2004) oder als ein System von Debatten und Praxen (Vgl. Rommelspacher 2009) verstanden werden. Daraus lässt sich folgern, dass Rassismus ein umfassendes Denksystem ist, welches nicht nur auf der individuellen, sondern in Anlehnung an Rommelspacher und Terkessidis ebenso auf der institutionellen sowie strukturellen Ebene funktioniert.

Seit der Einführung des Begriffs des institutionellen Rassismus in die breite Öffentlichkeit durch Carmichael/ Hamilton (1968) in deren Schrift „Black

\footnotetext{
${ }^{4}$ Der Prozess der Rassifizierung beschreibt die soziale Konstruktion von „Rasse“ und „Ethnizität“ (Vgl. Terkessidis 2004).

${ }^{5}$ Aufgrund des hier begrenzten Umfangs fokussiert sich dieser Aufsatz auf den in der breiten öffentlichen Aufmerksamkeit stehenden NSU-Untersuchungsausschuss im Bundestag - nicht zuletzt, da dieser das gesamte Geschehen in den Blick nahm. Die drei bereits abgeschlossenen Untersuchungsausschüsse der Landtage in Bayern, Sachsen und Thüringen werden im Rahmen dieses Beitrags nur kurz erwähnt. In die Analyse nicht miteinbezogen werden außerdem: Der Prozess am OLG München (Gerichtsverfahren gegen Beate Zschäpe sowie vier mutmaßliche Unterstützer: André Eminger, Holger Gerlach, Carsten S. und Ralf Wohlleben), die Bund-Länder-Kommission Rechtsterrorismus, die Thematisierung in verschiedenen Innenausschüssen der Landtage, kleine und große Anfragen im Bundestag und den Landtagen sowie die noch laufende Aufarbeitung der Untersuchungsausschüsse in Baden-Württemberg, Hessen, Nordrhein-Westfalen und der Neueinsetzung in Thüringen.
} 
Power", wurde dieser in den darauffolgenden Jahren auf verschiedene strukturelle Bereiche, wie den Arbeits- und Wohnungsmarkt, angewandt, erweitert und theoretisch ausdifferenziert (Vgl. Knowles/Prewitt 1969. Benokraitis/Feagin 1977). Den frühen Theorien des institutionellen Rassismus war weitgehend gemeinsam, dass sie sich auf die (Re-)Produktion von rassialisierter Ungleichheit in einem breiten Spektrum von Institutionen oder Strukturen fokussierten (Vgl. Williams 1985).

Gegenüber dem Begriff und seiner Konzeptualisierung wurde jedoch seit jeher auch vielseitige Kritik geäußert. Diese kann im Wesentlichen in drei Aspekte unterteilt werden: (1) Die soziologische Überdehnung des Rassismusbegriffs (Vgl. Williams 1985. Miles 1992), (2) die Außerachtlassung des sozialen Kontextes und die fehlende Kontextualisierung (Vgl. Williams 1985. Essed 1991. Phillips 2011) sowie (3) die Verwischung zwischen Intentionalität und Nicht-Intentionalität und die fehlende Unterscheidung zwischen Überzeugungen und Handlungen (Vgl. Williams 1985. Miles 1992. Gomolla 2010).

Aufgrund dieser Ungenauigkeiten wurde die Analyse von institutionellem Rassismus in vielen Fällen daher der Komplexität nicht gerecht. So hat die soziologische Überdehnung teilweise dazu geführt, dass dem Begriff des institutionellen Rassismus die analytische Schärfe abhandengekommen ist oder wesentliche Einflussfaktoren wie soziale Herkunft und Geschlecht außer Acht gelassen wurden, weil institutioneller Rassismus als alleinige Erklärung für gesellschaftliche Ungleichheitsverhältnisse herangezogen wurde. Darüber hinaus erscheint es wichtig, die Verschränkung von rassistischen Formationen, die auf verschiedenen Ebenen stattfinden, zu berücksichtigen, da sich beispielsweise gesellschaftliche Verhältnisse in den Institutionen artikulieren (Vgl. Karakayalı/ Tsianos 2004).

Bei der Verwendung des Begriffs des institutionellen Rassismus sollte folglich darauf geachtet werden, ihn in seiner Bedeutung nicht $\mathrm{zu}$ überdehnen, den Begriff für den jeweiligen Fall zu kontextualisieren, indem die gegebenen institutionellen Rahmenbedingungen beschrieben werden, und ihn auf einer bestimmten Analyseebene zu verorten (Vgl. Singh 2000). Wird der Begriff des institutionellen Rassismus entsprechend analytisch ausschließlich für eine institutionelle Mesoebene verwendet, so lässt er sich in den Organisationsstrukturen einer Gesellschaft verorten. Der Begriff impliziert folglich nicht, dass alle Mitarbeiter_innen einer Organisation rassistisch motiviert denken oder handeln oder bestimmt ein Maß, inwieweit rassistische Denk- und Handlungsmuster auf individueller Ebene vorhanden sind.

Die Mechanismen des institutionellen Rassismus werden in den Organisationstrukturen laut Hall ,auf informellen und unausgesprochenen Wegen durch ihre Routinen und täglichen Verfahren als ein unzerstörbarer Teil 
des institutionellen Habitus weitergegeben (Vgl. Hall 2001). Auf diese Weise wird diese Art von Rassismus „Routine, gewohnt, selbstverständlich“ (ebd., 165). Jedoch ist zu beachten, dass die Organisationsebene im wechselseitigen Austausch mit der individuellen Mikroebene sowie der gesellschaftlichen Makroebene steht und auf diese Weise ebenfalls in einem bestimmten Zeitverlauf gewisse Anpassungsprozesse durchläuft, da sich beispielsweise rassistische Diskurse auf der Makroebene in Praktiken auf der Organisationsebene übersetzen, Gesetze Veränderungen in bestimmten Abläufen hervorrufen oder sich individuelles Verhalten unter bestimmten Umständen auf die Organisationskultur auswirken kann.

\section{Kontextualisierung: Institutioneller Rassismus in Ermittlungs- und Sicherheitsbehörden}

Um den Begriff des institutionellen Rassismus für die Organisationsebene im Allgemeinen und insbesondere für die Analyse von Ermittlungs- und Sicherheitsbehörden anzuwenden und zu spezifizieren, empfiehlt es sich, das Konzept des rassistischen Wissens miteinzubeziehen. Terkessidis (1998) entwickelte dieses als Ausdruck, der Rassismus nicht als Vorurteil, sondern als Teil eines gesellschaftlichen Wertesystems beschreibt, weiter. Er bezeichnet dieses Wissen nicht als Vorurteil, sondern als hegemoniale Wissensform, da es sich um einen verbreiteten gesellschaftlichen Wissensbestand handelt. Auch innerhalb der Institutionen der Ermittlungs- und Sicherheitsbehörden hat sich ein bestimmter (kultur-)rassistischer Wissensbestand etabliert, der sich in bestimmte routinierte Abläufe, Praktiken und Verfahren übersetzt.

Als heutiges Paradigma des rassistischen Wissens lässt sich der durch die kulturalistische Wende entstandene differentielle Rassismus beschreiben (Vgl. Balibar/Wallerstein 1988. Terkessidis 1998). Im differentiellen Rassismus ist die Homogenisierung, Rassifizierung und Hierarchisierung im Wesentlichen von einem Kulturrassismus geprägt (Vgl. Bojadžijev 2008). In der kontextuellen Einbettung von (kultur-)rassistischen Wissensbeständen in Hinblick auf Ermittlungs- und Sicherheitsbehörden äußern sich diese exemplarisch in institutionalisierten Praktiken des sogenannten Racial Profiling, der Kriminalisierung von rassifizierten Personen sowie weiteren Praktiken und Verfahren, die auf institutionalisierte Wissensbestände der (kultur-)rassistischen Homogenisierung, Rassifizierung und Hierarchisierung basieren. 


\subsection{Institutioneller Rassismus im Fall Stephen Lawrence: Die verschleppte Aufklärung}

Am Abend des 22. April 1993 befand sich der 18-jährige, Schwarze Jugendliche Stephen Lawrence in Begleitung seines gleichaltrigen Freundes Duwayne Brooks auf dem Nachhauseweg. An einer Bushaltestelle in Eltham, einem Stadtteil im Südosten Londons, wurde Stephen Lawrence von einer Gruppe weißer Jugendlicher aus rassistischen Tatmotiven niedergestochen und erlag noch vor Ort seinen Verletzungen. Als die ersten Polizist_innen am Tatort eintrafen, wurden die beiden Schwarzen Jugendlichen weder als Opfer eines Angriffs behandelt noch wurde eine angemessene erste Hilfe geleistet (Vgl. Bowling/Phillips 2002). Zwar wurden nach kurzer Zeit Verdächtige festgenommen, aufgrund eines unsachgemäßen Umgangs mit den Beweisen wurden diese jedoch wieder freigelassen. Die Verbindungsbeamten verhielten sich gegenüber den Eltern von Stephen Lawrence unsensibel und unprofessionell. Auch wurden die Eltern über den Fortgang der Ermittlungen unzureichend informiert. Doreen Lawrence, die Mutter von Stephen Lawrence beschrieb den Umgang wie folgt: „It was a patronising way in which they dealt with me and that came across as being racist" (Home Office 1999, Para 4.4).

Trotz eindeutiger Zeug_innenaussagen wurde das Verbrechen lange Zeit nicht als rassistisch motiviert eingestuft. Aufgrund der mangelhaften Beweislage und Dokumentation des Falls wurde dieser frühzeitig abgeschlossen und hatte keine Verurteilung der Täter zur Folge. Eine Privatklage der Familie führte zur Zulassung der Anklage gegen drei der Verdächtigen. Diese wurden jedoch aufgrund der mangelhaften Beweislage freigesprochen. Erst durch den Einsatz und eine Kampagne der Familienangehörigen konnten Ermittlungsfehler nachgewiesen und ein öffentlicher Untersuchungsausschuss unter der Leitung von Sir William Macpherson einberufen werden.

Die Kriminalisierung der Opfer - also die Annahme, die Opfer müssten auch Täter sein, um Opfer von Gewalt werden zu können (Vgl. Bowling/Phillips 2002) - und der unangemessene Umgang von Seiten der Ermittlungsbeamt_innen lassen sich auf (kultur-)rassistische Wissensbestände zurückführen. Durch etablierte Bilder von Banden und Kriminalität werden Schwarze Menschen in Großbritannien von der breiten Öffentlichkeit als Gefahr für die moralische Ordnung der Gesellschaft wahrgenommen. Es findet eine 
Rassifizierung von Gewalt und Kriminalität statt (Vgl. Patel/Tyrer 2011). Diese Bilder von der Gesetzlosigkeit Schwarzer Menschen, die schon lange Teil eines rassistischen Wissensbestands sind, kamen in ihrer modernen Form mit der moralischen Panik ${ }^{6}$ (bitte kurz erläutern, was das ist) der 1970er und 1980er auf (Vgl. Gilroy 1987) und übersetzten sich auch in die Arbeit, Routinen und Praktiken der Ermittlungsbehörden.

\subsection{Institutioneller Rassismus im Fall NSU: Die Rassifizierung von Kriminalität}

Analog zum aufgezeigten institutionellen Rassismus im Fall Stephen Lawrence lassen sich auch im NSU-Komplex die Kriminalisierung von Opfern, (kultur-) rassistische Wissensbestände und in dessen Folge eine Nichterkennung rassistischer Tatmotive ausmachen. So beschreibt Semiya Şimşek, die Tochter des ersten Mordopfers des NSU, dass ihr Vater im Rahmen der Ermittlungen verdächtigt wurde, mit Rauschgift gehandelt oder diese zumindest transportiert zu haben.Dieser Verdacht hielt mehr als ein Jahrzehnt an (Vgl. Şimşek/Schwarz 2013). Als die Ermittlungen nicht weiterführten, erzählten die Beamten der Witwe des Opfers die frei erfundene Geschichte, ihr Mann habe noch eine zweite Familie gehabt: Eine deutsche, blonde Frau, von der sie auch Fotos zeigten und mit der er ebenfalls zwei Kinder habe (Vgl. ebd.). Auf diese Weise sollte ein bestimmtes Verhalten der Witwe provoziert werden, um an bislang als zurückgehalten vermutete Informationen zu kommen.

Durch die Namensgebung der Sonderkommission „Halbmond“ wie auch der Besonderen Aufbauorganisation „Bosporus“ wird ersichtlich, dass die kriminellen Taten deutlich außerhalb der weißen, deutschen Mehrheitsbevölkerung, und sogar außerhalb des Landes verortet wurden. Nebenklagevertreter_innen im NSU-Prozess am Oberlandesgericht München fassten Folgendes zusammen: „Hunderte Zeuginnen und Zeugen wurden selbst in der Türkei verhört, Drogenhunde eingesetzt, fingierte Dönerbuden eröffnet, verdeckte Ermittler als Journalisten eingesetzt, Steuerbanderolen auf Zigarettenschachteln überprüft; selbst ein Wahrsager wurde befragt. Über elf Jahre fahndeten hunderte Ermittler in die falsche Richtung. Alle Zeugen, alle Analysen, alle Beweismittel, die auf rassistisch motivierte Anschläge hindeuteten, wurden konsequent ignoriert" (von der Behrens et al. 2013, o.S.).

\footnotetext{
${ }^{6}$ Als moral panic bezeichneten Hall et al. (1978) die Diskrepanz zwischen tatsächlicher Bedrohung durch die muggings in den 1970er Jahren in den USA und der Härte der darauffolgenden Reaktionen, die dazu führten, dass Schwarze Jugendliche unter ständigem Verdacht der Polizei standen.
} 
Um ein Täterprofil zu zeichnen, wurden im Laufe der Ermittlungen mehrere sogenannte Operative Fallanalysen (OFA) erstellt. Verwies die erste OFA im Jahr 2005 auf die sogenannte Organisationstheorie, brachte die zweite OFA im Mai 2006 zum ersten Mal die Alternativhypothese eines möglicherweise rassistisch motivierten Einzeltäters hervor. Am Tag der Vorstellung der zweiten OFA wurde jedoch eine weitere OFA beim LKA Baden-Württemberg in Auftrag gegeben, da die Fixierung auf das Umfeld der Opfer und den Gedanken der Organisierten Kriminalität vorherrschend war (Vgl. BT-Drs. 17/14600).

Diese dritte OFA, die im Januar 2007 erschien, liefert wohl die eindringlichsten Beispiele für (kultur-)rassistische Wissensbestände auf der institutionellen Ebene. So heißt es dort wörtlich: „Aufgrund der Tatsache, dass man 9 türkischsprachige Opfer hat, ist nicht auszuschließen, dass die Täter über die türkische Sprache den Bezug zu den Opfern hergestellt haben und die Täter demzufolge ebenfalls einen Bezug zu dieser Sprache haben. Auch spricht der die Gruppe prägende rigide Ehrenkodex eher für eine Gruppierung im ost- bzw. südosteuropäischen Raum (nicht europäisch westlicher Hintergrund)“ (BT-Drs. 17/14600, S. 878).

Weiter heißt es in der OFA: „Vor dem Hintergrund, dass die Tötung von Menschen in unserem Kulturraum mit einem hohen Tabu belegt ist, ist abzuleiten, dass der Täter hinsichtlich seines Verhaltenssystems weit außerhalb des hiesigen Normen- und Wertesystems verortet ist" (ebd).

Die Morde wurden folglich ,an der Schnittstelle von antimigrantischen und antimuslimischen Diskursen“" (Attia 2014, S. 129) als Taten von Eingewanderten eingestuft. So folgten die Ermittlungen „diesem gesellschaftlich wirkmächtigen Wissen über „die Anderen“, was dazu führte, dass die Täter_innen lange unerkannt bleiben und ihre Mordserie fortsetzen konnten, während die Hinterbliebenen beschuldigt wurden, an den Morden beteiligt zu sein" (ebd., Seitenzahl).

Es lässt sich zusammenfassend feststellen, dass institutioneller Rassismus - als eine Ursache unter mehreren ${ }^{7}$ (Vgl. Aust/Laabs 2014. Gensing 2012. Förster 2014) - dazu beigetragen hat, dass die Mordserie des Nationalsozialistischen Untergrunds in dessen aktiver Zeit von Ermittlungs- und Sicherheitsbehörden nicht aufgedeckt wurde und sich in Form von bestimmten institutionalisierten

\footnotetext{
${ }^{7}$ Das Versagen der Ermittlungs- und Sicherheitsbehörden im NSU-Komplex ist sehr vielschichtig, da es sich um ein organisiertes, rechtsterroristisches Netzwerk und eine Vielzahl an Straftaten handelt. Außerdem erstreckte sich der zeitliche Rahmen, in dem die NSU-Mordserie und die weiteren Straftaten verübt wurden, über mehrere Jahre, die Verbrechen fanden bundesweit statt und fallen somit in unterschiedliche Zuständigkeiten der Ermittlungs- und Sicherheitsbehörden in Bund und Ländern. Das Versagen der Sicherheitsarchitektur ist somit auf vielerlei Ursachen zurückzuführen.
} 
(kultur-)rassistischen Wissensbeständen und daraus resultierenden Ermittlungspraktiken äußerte.

\section{Zwischen Bekenntnis und Vermeidung:Identifizierung und Thematisierung von institutionellem Rassismus in Deutschland und Großbritannien}

Sowohl im Fall Stephen Lawrence wie auch im Fall NSU führte die nachholende Aufarbeitung zur Einsetzung von Untersuchungsausschüssen. In Großbritannien wurde im Jahr 1999 die öffentliche Untersuchungskommission unter der Leitung des ehemaligen Richters des Obersten Gerichtshofs Sir William Macpherson of Cluny einberufen und in Deutschland setzte der Deutsche Bundestag den parlamentarischen NSU-Untersuchungsausschuss ein. In beiden Fällen stellt sich die Frage, inwiefern sie institutionellen Rassismus - als eine Ursache unter mehreren - für die Nichtaufklärung der Straftaten identifizierten und thematisierten.

\subsection{Der Macpherson Report als Wendepunkt für die Thematisierung von institutionellem Rassismus in Großbritannien}

Der Umstand des Mordes an Stephen Lawrence, die fehlende Verurteilung der Täter und die weit verbreitete Wahrnehmung, dass die Untersuchung des Falls von der Metropolitan Police nicht angemessen gehandhabt wurde, veranlasste die Familie von Stephen Lawrence zur Initiierung einer Kampagne. Diese wiederum führte zur Einsetzung der Macpherson-Kommission im Jahr 1997 (Vgl. Foster/Newburn/Souhamie 2005). Ihr formulierter Auftrag war der Folgende: „To identify the lessons to be learned for the investigation and prosecution of racially motivated crimes" (Home Office 1999, Para 3.1). Der Fokus der Untersuchung hatte zum einen das Ziel, die Umstände des Mordes an Stephen Lawrence, die Reaktion der Polizei sowie der nachfolgenden Ermittlung aufzuklären und zum anderen die Themen im Hinblick auf das Verhältnis zwischen Polizei und rassifizierten Personen auf einer überregionalen und gesellschaftlichen Ebene zu erfassen (Vgl. McLaughlin/Murji 1999).

Die Untersuchung fand unter weitreichender Einbeziehung verschiedener gesellschaftlicher Gruppen und einer umfassenden öffentlichen Diskussion über institutionellen Rassismus statt. Am 24. Februar 1999 veröffentlichte die 
Kommission ihre Schlussfolgerungen in einem 335-seitigen Dokument, dem sogenannten Macpherson Report. Der britische Bericht arbeitet institutionellen Rassismus als Ursache für die Nichtaufklärung detailliert heraus und stellt die Thematisierung dessen in den Vordergrund, so dass der Bericht und die Aufklärung auch fortwährend damit assoziiert wurden. Die Kommission fasste zusammen: „The investigation was marred by a combination of professional incompetence, institutional racism and a failure of leadership by senior officers"(Home Office 1999, Para 64.1).

Institutioneller Rassismus wurde hierbei definiert als: „The collective failure of an organisation to provide an appropriate and professional service to people because of their colour, culture, or ethnic origin. It can be seen or detected in processes, attitudes and behaviour which amount to discrimination through unwitting prejudice, ignorance, thoughtlessness and racist stereotyping which disadvantage minority ethnic people“ (ebd., Para 6.34).

Zur Veröffentlichung des Macpherson Reports äußerte sich der damalige Premierminister Tony Blair: „The publication of today's report on the killing of Stephen Lawrence is a very important moment in the life of our country. It is a moment to reflect, to learn and to change. It will certainly lead to new laws but, more than that, it must lead to new attitudes, to a new era in race relations, and to a new more tolerant and more inclusive Britain" (Hansard 1999, col. 380). Diese Aussage unterstreicht den politischen Willen, der hinter den Empfehlungen stand.

Der Bericht enthält 70 detaillierte Empfehlungen für eine umfassende Reform der Strafjustiz und des Bildungssystems. Die Vorschläge umfassten die Ausbildung und die interkulturelle Öffnung der Institutionen der Polizei, die Benennung von Rassismus als Tatmotiv, die Verbesserung im Umgang mit den Familien, Verbesserung in der Ersten Hilfe und die Dokumentation von Stop and Searches (Vgl. Home Office 1999). Die im Bericht enthaltenen Empfehlungen führten in Großbritannien zur umfangreichsten Reform im Hinblick auf das Verhältnis zwischen der Polizei und ethnisierten Minderheiten bzw. rassifizierten Personen (Vgl. Bowling/Phillips 2002). So wurden neue Aufsichtsgremien eingerichtet, Kontrollmechanismen eingeführt, Richtlinien für den Tatbestand einer rassistisch motivierten Gewalttat formuliert und die Antidiskriminierungsgesetze maßgeblich erweitert (Vgl. Lewicki 2016 in diesem Band).

Eine staatliche Evaluation des Fortschritts, die zehn Jahre nach Erscheinen des Berichts durchgeführt wurde, stellte heraus, dass 67 der 70 Empfehlungen teilweise oder komplett umgesetzt wurden. Jedoch besteht weiterhin ein großer Handlungsbedarf: So besteht weiterhin eine große Disproportionalität in den Stop-and-Search-Statistiken und die Zielquote von 7 Prozent für die 
„interkulturelle Öffnung“ in der Rekrutierung wurde ebenfalls nicht erreicht. Hier konnte der Anteil rassifizierter Personen von 1999 bis 2009 lediglich von 2 auf 4,1 Prozent gesteigert werden (Vgl. House of Commons Home Affairs Committee 2009).

Zwar wird der Macpherson Report oftmals als zentrales Beispiel für die Benennung und Dokumentation von institutionellem Rassismus angeführt, jedoch weist die Definition einige der bereits in Kapitel 2 beschriebenen konzeptionellen Schwächen des Begriffs auf (Vgl. Gomolla 2010). Im Wesentlichen konzentriert sich die Kritik hierbei auf die Verschmelzung von individuellem und institutionellem Rassismus bzw. der fehlenden Differenzierung zwischen institutionellem Rassismus als Ergebnis und Ursache (Vgl. Bourne 2001. Bridges 2000. Lea 2000. Solomos 1999). Möglicherweise ist dies auch ein Grund dafür, dass institutioneller Rassismus im Zuge der Veröffentlichung des Berichts von Polizist_innen vehement zurückgewiesen wurde, da die Bedeutung so interpretiert wurde, als sei die Polizei durchwegs von individuellem Rassismus gekennzeichnet (Vgl. Foster/Newburn/Souhamie 2005).

Zusammenfassend lässt sich feststellen, dass die Thematisierung von institutionellem Rassismus im Macpherson Report an zentraler Stelle stand. Die vage Definition führte jedoch dazu, dass Ursachen und Verantwortung unklar benannt wurden und sich dies für die Akzeptanz innerhalb der Institutionen sowie für die Umsetzung der Reformvorschläge als hinderlich erwies. Dennoch stellt die Aufarbeitung des Macpherson Reports - nicht zuletzt aufgrund des Einbezugs der Öffentlichkeit in die Ausschussarbeit - einen zentralen Wendepunkt in der Identifizierung und Thematisierung von institutionellem Rassismus in der britischen Gesellschaft dar.

\subsection{Der NSU-Untersuchungsausschuss im Bundestag: Fehlende gemeinsame Sprache}

Der Deutsche Bundestag setzte am 26. Januar 2012 einen Untersuchungsausschuss zur Aufklärung der Hintergründe und Zusammenhänge des NSU-Komplexes ein. Es war der erste Untersuchungsausschuss in der Geschichte des Bundestags, der auf gemeinsamen Beschluss aller Fraktionen einberufen wurde. Dies unterstrich die gesellschaftspolitische Relevanz, die der Aufarbeitung beigemessen wurde. Am 22. August 2013 - also nach 19 Monaten Ausschussarbeit - wurde der Abschlussbericht im Bundestag vorgelegt. In seinen Bewertungen wurden Schlussfolgerungen für die zukünftige Arbeit der Sicherheits- und Ermittlungsbehörden gezogen. Diesbezüglich fanden eine 
überfraktionelle, gemeinsame Bewertung, die 47 Empfehlungen umfasst, sowie fraktionsinterne Stellungnahmen, die den Fraktionen Raum boten, ihre eigenen Schwerpunkte herauszuarbeiten, statt.

\subsubsection{Vage Formulierungen in der gemeinsamen Bewertung}

Der gemeinsamen Bewertung des Untersuchungsausschusses im Bundestag schließen sich 47 fraktionsübergreifend einstimmig beschlossene Empfehlungen an. Im Hinblick auf die Frage, ob es in der parlamentarischen Aufarbeitung des NSU-Komplexes zu einer eindeutigen Identifizierung und Thematisierung von institutionellem Rassismus gekommen ist, werden hier insbesondere die Empfehlungen für die Organisationsbereiche der Polizei- und Verfassungsschutzbehörden betrachtet.

Für den Bereich der Polizei sind vier zentrale Empfehlungen vorzufinden: Erstens soll sich die Vielfalt der Gesellschaft in der Rekrutierung und Personalstruktur widerspiegeln, zweitens soll die interkulturelle Kompetenz als fester und verpflichtender Bestandteil der Aus- und Fortbildung vorgesehen werden, drittens soll die Kommunikation mit Opfern und ihrem näheren Umfeld durch spezielle Vermittlungsbeamt_innen verbessert werden und viertens sollen Opferzeugen verpflichtend auf ihre bestehenden Rechte verwiesen werden. Für den Bereich der Verfassungsschutzbehörden finden sich zwei ähnliche Empfehlungen: Zum einen soll hinsichtlich einer Sensibilisierung für Gefahren des Rechtsextremismus und mehr Offenheit in den Institutionen ein Mentalitätswandel innerhalb des Verfassungsschutzes erzielt und zum anderen die ,interkulturelle Öffnung“ der Verfassungsschutzbehörden vorangetrieben und eine Fehlerkultur etabliert werden(Vgl. BT-Drs. 17/14600). ${ }^{8}$

Es wird ersichtlich, dass hinsichtlich der Empfehlungen für den Bereich der Polizei nicht direkt auf institutionellen Rassismus hingewiesen wird. Auch sind die Empfehlungen sehr vage gehalten, so werden beispielsweise keine Kennzahlen für die ,interkulturelle Öffnung“ der Rekrutierung festgelegt und interkulturelle Kompetenz nicht näher definiert. Für den Bereich des Verfassungsschutzes wurden ebenfalls Defizite in der Arbeitskultur sowie in Wahrnehmungs- und Handlungspraktiken festgestellt. Eine klare Benennung oder inhaltliche Tiefe der Empfehlungen bleibt ebenfalls aus.

Es lässt sich festhalten, dass vom Untersuchungsausschuss im Bundestag erkannt und benannt wurde, dass die Institutionen der Polizei und des

\footnotetext{
8 Die Empfehlungen 11 bis 14 für den Bereich der Polizei sowie 38 und 40 für die Verfassungsschutzbehörden wurden auf den Sinngehalt verdichtet und gekürzt. Für den genauen Wortlaut siehe BT-Drs. 17/14600, S. 862-865.
} 
Verfassungsschutzes Nachholbedarf hinsichtlich eines angemessen und den Umständen einer Migrationsgesellschaft entsprechenden Verhaltens gegenüber Vielfalt aufweisen. Ob mit der Einführung des Begriffes der interkulturellen Kompetenz oder des vorgeschlagenen Konzepts der „,interkulturellen Öffnung“ der Abbau von (kultur-)rassistischen Wissensbeständen gemeint ist, bleibt Spekulation. Die Empfehlungen bleiben undefiniert, vage und bieten viel Spielraum für mögliche Auslegungen.

\subsubsection{Detaillierte Analysen in den ergänzende Stellungnahmen der Fraktionen}

Im Rahmen des NSU-Untersuchungsausschusses im Bundestag wurde allen Fraktionen die Möglichkeit eingeräumt, ergänzende Stellungnahmen abzugeben, sofern sie die gemeinsam verabschiedeten Bewertungen als unzureichend empfanden. Während die CDU/CSU-Fraktion keinen weiteren Bedarf für Ergänzungen sah und auf die Einbringung weiterer Aspekte in ihrer ergänzenden Stellungnahme verzichtete und die FDP-Fraktion sich auf andere Themengebiete fokussierte, wies die SPD-Fraktion für die Notwendigkeit ihres Einzelvotums gleich zu Beginn ihrer Stellungnahme darauf hin, dass im gemeinsamen Bericht aus ihrer Sicht ,nicht ausreichend deutlich [wird], dass routinisierte Verdachtsund Vorurteilsstrukturen und unbewusste Prozesse institutioneller Diskriminierung ganz wesentlich für die andauernde Betriebsblindheit der Ermittler bezüglich eines möglichen rassistischen Hintergrunds der Mordserie verantwortlich waren" (ebd., S. 872). Des Weiteren wurde betont, dass „strukturelle rassistische Vorurteile (...) eine wesentliche Ursache für die fehlende Offenheit der Ermittlungen zu den Morden und Sprengstoffattentaten des NSU“ (ebd., S. 877) waren. Hierfür wurden die zentralen Beispiele aus den fehlgeleiteten Untersuchungen angeführt: Einseitiger Fokus auf das Umfeld der Opfer sowie Vermutung einer Beziehungstat, Betäubungsmittelkriminalität, Schutzgelderpressungen, angebliche Spielschulden, kriminelle Organisation oder Ausländerkriminalität. Als weitere Beispiele für die beschriebenen „,vorurteilsbehaftete[n] Routinen in der Polizeiarbeit, die Delinquenz bestimmten Personengruppen, Milieus und Ethnien schematisch zuordnen“ (ebd., S. 879), wurden die Namensgebung von eingesetzten Ermittlungseinheiten, der Einsatz verdeckter Ermittler, das dokumentierte Misstrauen gegenüber dem Opferumfeld sowie weitere Ermittlungspraktiken angeführt. Es wird auf ,verbreitete Wissensbestände“" (ebd.) verwiesen, auf die zurückgegriffen wurde, weil keine Selbstreflexion stattgefunden habe. Die SPD-Fraktion hielt in ihren Ergänzungen zum NSU-Untersuchungsbericht fest, dass Rassismus ,nicht einfach per se 
verleugnet werden“ (ebd., S. 880) dürfe und er ,als strukturelles Problem auch im institutionellen Kontext anerkannt werden“ (ebd.) müsse. Als Empfehlungen für eine Verbesserung der zukünftigen Ermittlungsarbeit wurden Maßnahmen zur Stärkung der interkulturellen Kompetenz, zur Bekämpfung institutioneller Vorurteilsstrukturen, Verbesserung der Personalauswahl, eine unabhängige Untersuchung zu Vorurteilen in der Polizei und deutliche Verbesserung in Ausund Fortbildung vorgeschlagen.

DIE LINKE wies ebenfalls bereits $\mathrm{zu}$ Beginn ihrer fraktionsinternen Stellungnahme darauf hin, dass die Erklärung für die einseitige Ermittlungsrichtung, ,die die Ermordeten, ihre Angehörigen und die Opfer der Sprengstoffanschläge kriminalisierte und stigmatisierte“ (ebd., S. 983), institutioneller Rassismus sei. Sie wies explizit darauf hin, dass es sich hierbei nicht um die individuellen Einstellungen einzelner Ermittler_innen handle, sondern um ein „strukturelles Merkmal der Polizeiarbeit bei den Ermittlungen zur rassistischen Mordserie“ (ebd.). Des Weiteren wurde der Begriff des institutionellen Rassismus als „eine Form des Rassismus, die von Institutionen der Gesellschaft, ihren Verfahren, Normen und rechtlichen Grundlagen ausgeht und zunächst unabhängig von der Motivation der darin handelnden Individuen ist“" (ebd., S. 989), näher definiert. Die Fraktion DIE LINKE verwies auf institutionellen Rassismus bei den Ermittlungen zur NSU-Mordserie und den Sprengstoffanschlägen in Köln und benannte hierbei als Beispiele die Operative Fallanalyse des LKA Baden-Württemberg, ethnisierende Zuschreibungen, Antiziganismus bei den Ermittlungen im Mordfall Kiesewetter und die Ermittlungen im Bereich Organisierte Kriminalität. Anschließend empfahl die Fraktion für den Bereich der Polizei unabhängige Polizeibeschwerdestellen bzw. Polizeibeobachtung und erhebliche Verbesserungen in den Bereichen der Ausund Fortbildung sowie bei der ,interkulturellen Öffnung“ hinsichtlich der Rekrutierung. Auch wurde auf die Notwendigkeit einer Einstellungsbefragung zum Thema „Rassismus und Polizei“ hingewiesen, um „die Diskussion über möglicherweise vorhandene rassistische Vorurteile und Einstellungspotenziale in den Polizeien auf eine sachliche Grundlage“ (ebd., S. 984) zu stellen und notwendige Maßnahmen und Empfehlungen entwickeln zu können.

Die Fraktion BÜNDNIS 90/DIE GRÜNEN benannte in ihren Empfehlungen für den Bereich der Polizei und Staatsanwaltschaften die Existenz einer offensichtlichen „Vorurteilstruktur innerhalb der Polizei, welche die Fehl- oder Nichtermittlungen (...) begünstigt oder bedingt" (ebd., S. 1036) habe. So haben die Untersuchungen des Untersuchungsausschusses aufgezeigt, dass diese Fehloder Nichtermittlungen hinsichtlich der NSU-Mordserie „mit rassistischen Vorurteilen in Zusammenhang standen“ (ebd.). Als Beispiele hierfür wurden die Benennungspraxis der polizeilichen Organisationseinheiten nach „Halbmond“ 
und „Bosporus“ wie auch die Operative Fallanalyse des LKA BadenWürttemberg angeführt. In der Erkenntnis kam die Fraktion daher zu ähnlichen Schlüssen wie die SPD-Fraktion und die Fraktion DIE LINKE, blieb jedoch weitaus zurückhaltender in ihrer Formulierung als die anderen beiden. Als Empfehlung führten sie ebenfalls „eine unabhängige, empirische Untersuchung $\mathrm{zu}$ Vorurteilen und Diskriminierungsstrukturen innerhalb der deutschen Sicherheits- und Strafverfolgungsbehörden“ (ebd., Seitenzahl) an und zudem die Verankerung von interkultureller Kompetenz im Leitbild der Polizei, mit dem Hinweis darauf, dass sich diese auch in der alltäglichen Praxis der Polizeiarbeit niederschlagen müsse.

\section{Fazit}

Die Betrachtung des Falls Stephen Lawrence und des NSU-Komplexes hat gezeigt, dass in beiden Fällen institutioneller Rassismus in den Ermittlungs- und Sicherheitsbehörden - als eine Ursache unter mehreren - dazu beigetragen hat, dass die Aufklärung der Taten verhindert oder zumindest erschwert wurde. Als die wesentlichen Abläufe und Praktiken, die sich aufgrund (kultur-)rassistischer Wissensbestände institutionalisiert haben und anhand derer sich ein institutioneller Rassismus feststellen ließ, konnten die Kriminalisierung der Opfer, der Umgang mit den Angehörigen sowie die fehlende Überprüfung eines rassistischen Tatmotivs bestimmt werden.

Auch wenn die Identifizierung von institutionellem Rassismus in beiden Fällen stattgefunden hat, wurde der Thematisierung jedoch in der Aufarbeitung des NSU-Untersuchungsausschusses aufgrund von interfraktionellen Differenzen kein sichtbarer Platz in der gemeinsamen Bewertung eingeräumt, weswegen sie im Gegensatz zum Macpherson Report auch keine breite öffentliche Aufmerksamkeit erfuhr und es in Deutschland nicht zu einer Debatte über institutionellen Rassismus kam.

Die Analyse des Untersuchungsausschusses im Bundestag hat ergeben, dass in den gemeinsamen Bewertungen ein großer Nachholbedarf in den Institutionen der Polizei und des Verfassungsschutzes hinsichtlich eines angemessenen Umgangs mit Vielfalt konstatiert wurde. So bilden diese Institutionen bislang weder die Vielfalt der Gesellschaft $a b$, noch sind deren Haltung und Handlungspraktiken darauf ausgelegt, dies zu tun. Die Empfehlungen deuten auch darauf hin, dass die Institutionen sich deutlich verändern müssen, dennoch bleiben sie äußerst vage in ihrer Ausgestaltung. In der Analyse zeigte sich, dass in den gemeinsamen Bewertungen des Abschlussberichts nicht eindeutig auf 
institutionellen Rassismus und (kultur-)rassistische Wissensbestände hingewiesen wurde.

Deutlichere Ergebnisse lieferte die Analyse der fraktionsinternen Stellungnahmen. Die Fraktionen der SPD, DIE LINKE und BÜNDNIS 90/DIE GRÜNEN verwiesen in ihrem größten gemeinsamen Nenner auf offensichtliche Vorurteilsstrukturen in den Ermittlungs- und Sicherheitsbehörden, die eine Aufklärung der Straftaten des NSU verhindert oder zumindest erschwert haben. An verschiedenen Stellen wurde auch explizit darauf hingewiesen, dass institutionelle Routinen, Praktiken und Verfahren, die auf (kultur-)rassistischen Wissensbeständen basieren, zu einer Kriminalisierung der Opfer, einem unangemessenen Umgang mit deren Angehörigen sowie einer kaum hinterfragten und sich normalisierenden Ermittlung in Richtung Organisierter Kriminalität geführt haben. Es lässt sich jedoch festhalten, dass institutioneller Rassismus vor allem als Ergebnis und nur partiell als Ursache erkannt wurde. Auch lassen die fraktionsinternen Stellungnahmen eine große Varianz erkennen, weswegen sich in der parlamentarischen Untersuchung des NSU-Komplexes hinsichtlich der Thematisierung von institutionellem Rassismus kein einheitliches Bild abzeichnete und keine einheitliche Sprache gefunden werden konnte. Den fraktionsinternen Stellungnahmen wurde auch von politischer Seite keine starke Sichtbarkeit und der Thematisierung von institutionellem Rassismus im öffentlichen Diskurs bislang wenig Nachdruck verliehen.

Derzeit sieht es so aus, als habe Deutschland trotz all der bislang geleisteten parlamentarischen Aufarbeitungen seinen Stephen-Lawrence-Moment verpasst. Jedoch findet sich die Identifizierung von institutionellem Rassismus bereits in der Mehrheit der fraktionsinternen Stellungnahmen und die Aufarbeitung des NSU-Komplexes scheint ebenfalls noch lange nicht abgeschlossen zu sein. Es wird mehrfach darauf hingewiesen, dass die Aufklärung nun erst recht weiter angetrieben werden müsse (Vgl. Funke 2015). Aus verschiedenen Fraktionen wurden Forderungen formuliert, die entsprechenden Untersuchungsausschüsse aufgrund von offen gebliebenen Unklarheiten und Wissenslücken in den jeweils nächsten Legislaturperioden fortzuführen bzw. neueinzurichten - dies ist in Thüringen bereits geschehen und auch im Bundestag soll ab November 2015 ein neuer Untersuchungsausschuss eingerichtet werden (Vgl. Götschenberg 2015). Möglicherweise bietet diese weitere Vertiefung der Auseinandersetzung und Aufarbeitung die Chance für eine Fokusverschiebung hinsichtlich der Fehleranalyse sowie für eine eindeutigere Benennungspraxis, wie es auch bereits im Abschlussbericht des Thüringer Untersuchungsausschusses zu erkennen war (Vgl. THL-Drs. 5/8080) und dies seit längerer Zeit von zivilgesellschaftlichen Akteuren gefordert wird (Vgl. DIMR 2013. TGD 2013). Des Weiteren könnten die in den Bundesländern Baden-Württemberg, Hessen und Nordrhein-Westfalen 
eingerichteten Untersuchungsausschüsse, die zur Aufarbeitung des NSUKomplexes und der länderspezifischen Arbeit der Ermittlungs- und Sicherheitsbehörden eingesetzt wurden, diesen zentralen Aspekt in ihrer Fehleranalyse herausstellen.

Es wird in naher Zukunft auch $\mathrm{zu}$ untersuchen sein, wie die recht vage gehaltenen, fraktionsübergreifenden Empfehlungen des Untersuchungsausschusses auf Bundesebene, die darauf abzielen könnten, (kultur-)rassistischen Wissensbeständen und institutionalisierten Praktiken entgegenzuwirken in politische Maßnahmen und Gesetze überführt werden, so wie dies nach dem Macpherson Report in Großbritannien geschah. Aus dem Fall Stephen Lawrence lässt sich für die Aufarbeitung des NSU-Komplexes wie auch für die Identifizierung und gesellschaftspolitische Thematisierung von institutionellem Rassismus in Deutschland viel lernen. Jedoch sollte - und auch das lässt sich aus der Evaluation des Macpherson Reports erkennen - eine detaillierte Analyse und genaue Definition des Begriffs des institutionellen Rassismus die Grundlage dafür bilden, da in Großbritannien aufgrund konzeptioneller Schwächen in der Begriffsdefinition viele Reformen nicht ihre formulierten Ziele erreichten oder aufgrund von Unverständlichkeiten Widerstände aufkamen.

\section{Literatur}

Attia, Iman (2014): Rassismus (nicht) beim Namen nennen. In: Aus Politik und Zeitgeschichte, 13$14 / 2014$, S. 8-14.

Aust, Stefan/Laabs, Dirk (2014): Heimatschutz. Der Staat und die Mordserie des NSU, München: Pantheon Verlag.

Balibar, Etienne/Wallerstein, Immanuel (1988): Race, nation, class. Ambiguous Identities, London/ New York: Verso.

Bax, Daniel (27.10.2013): Racial Profiling bei der Polizei: „Igittigitt, das ist Rassismus“, Interview mit Rainer Wendt. In: Die Tageszeitung. Abrufbar unter: http://www.taz.de/!5056271/ (Stand: 10.10.2015).

Benokraitis, Nijole/Feagin, Joe (1977): Institutional Racism: A Perspective in Search of Clarity and Research, In: Charles V. Wille (Hrsg.): Black/Brown/White Relations, Race Relations in the 1970, New Jersey: Transaction Books, S. 121-143.

Bojadžijev, Manuela (2008): Die windige Internationale. Rassismus und Kämpfe der Migration, Münster: Westfälisches Dampfboot.

Bojadžijev, Manuela (2013): Wer von Rassismus nicht reden will. Einige Reflexionen zur aktuellen Bedeutung von Rassismus und seiner Analyse, In: Imke Schmincke und Jasmin Siri (Hrsg.): NSU Terror. Ermittlungen am rechten Abgrund. Ereignis, Kontexte, Diskurse, Bielefeld: transcript Verlag, S. 145-154.

Bourne, Jenny (2001): The life and times of institutional racism. In: Race and Class, 43 (2), S. 7-22.

Bowling, Ben/Phillips, Coretta (2002): Race, Crime and Justice, Harlow: Longman.

Bridges, Lee (2000): The Lawrence Inquiry - incompetence, corruption and institutional racism. In: Journal of Law and Society, 26 (3), S. 289-322. 
Carmichael, Stokely/Hamilton, Charles Vernon (1968): Black Power. Die Politik der Befreiung in Amerika, Stuttgart: Günther Verlag.

Deutscher Bundestag Drucksache 17/14600 (22.08.2013): Beschlussempfehlung und Bericht des 2. Untersuchungsausschusses nach Artikel 44 des Grundgesetzes. Abrufbar unter: http://dipbt.bundestag.de/dip21/btd/17/146/1714600.pdf (Stand: 10.10.2015).

Deutsches Institut für Menschenrechte (20.08.2013): Zur Veröffentlichung des Abschlussberichts des Untersuchungsausschusses des Bundestages zur „Terrorgruppe nationalsozialistischer Untergrund. Hintergrundpapier zur Pressemitteilung vom 20. August 2013. Abrufbar unter: http://www.institut-fuer-menschenrechte.de/fileadmin/user_upload/PDF-

Dateien/Sonstiges/hintergrundpapier_Zur_Veroeffentlichung_des_Abschlussberichts_des_Unter suchungsausschusses_des_Bundestages_zur_Terrorgruppe_NSU.p.pdf (Stand: 10.10.2015).

Eggers, Maureen Maisha/Kilomba, Grada/Piesche, Peggy/Arndt, Susan (Hrsg.) (2009): Mythen, Masken und Subjekte. Kritische Weißseinsforschung in Deutschland, Münster: Unrast Verlag.

Essed, Philomena (1991): Understanding Everyday Racism, London: Sage.

Foster, Janet/Newburn, Tim/Souhami, Anna (2005): Assessing the Impact of the Stephen Lawrence Inquiry. Home Office Research Study 294, London: Home Office.

Förster, Andreas (Hrsg.) (2014): Geheimsache NSU. Zehn Morde, von Aufklärung keine Spur, Tübingen: Klöpfer und Meyer.

Funke, Hajo (2015): Staatsaffäre NSU. Eine offene Untersuchung, Münster: Kontur-Verlag.

Gensing, Patrick (2012): Terror von rechts. Die Nazi-Morde und das Versagen der Politik, Berlin: Rotbuch Verlag.

Gilroy, Paul (1987): There Ain't No Black in the Union Jack, London: Hutchinson.

Gomolla, Mechtild (2010): Institutionelle Diskriminierung. Neue Zugänge zu einem alten Problem, In: Ulrike Hormel und Albert Scherr (Hrsg.): Diskriminierung. Grundlagen und Forschungsergebnisse, Wiesbaden: VS Verlag, S. 61-93.

Götschenberg, Michael (02.10.2015): Bundestagsfraktionen einig: Weg für neuen U-Ausschuss zum NSU frei. In: Tagesschau.de. Abrufbar unter: https://www.tagesschau.de/inland/nsu-u-ausschuss105.html (Stand: 10.10.2015).

Hall, Stuart (2001): Von Scarman zu Stephen Lawrence, In: Karen Schönwälder und Imke SturmMartin (Hrsg.) (2001): Die britische Gesellschaft zwischen Offenheit und Abgrenzung: Einwanderung und Integration vom 18. bis zum 20. Jahrhundert, Berlin/ Wien: Philo, S. 154168.

Hansard (24.02.1999): Parliamentary Debates, House of Commons. Abrufbar unter: http://www.publications.parliament.uk/pa/cm199899/cmhansrd/vo990224/debtext/9022420.htm, (Stand: 10.10.2015).

Home Office (1999): The Stephen Lawrence Inquiry: Report of an Inquiry by Sir William Macpherson of Cluny, Cm 4262-I, February 1999. Abrufbar unter: https://www.gov.uk/government/uploads/system/uploads/attachment_data/file/277111/4262.pdf (Stand: 10.10.2015).

House of Commons Home Affairs Committee (2009): The Macpherson Report - Ten Years On, London: The Stationery Office. Abrufbar unter: http://www.publications.parliament.uk/pa/cm200809/cmselect/cmhaff/427/427.pdf （Stand: 10.10.2015).

Karakayal1, Serhat/Tsianos, Vassilis (2004): Rassismus: Von Amts wegen anders. In: iz3W, Nr. 275, S. 14-17.

Kleffner, Heike/Feser, Andreas (18.11.2013): Dossier Rechtsextremismus: Der NSUUntersuchungsausschuss. In: Bundeszentrale für politische Bildung. Abrufbar unter: http://www.bpb.de/politik/extremismus/rechtsextremismus/172857/der-nsuuntersuchungsausschuss (Stand: 10.10.2015). 
Knowles, Louis L./Prewitt, Kenneth (1969): Institutional Racism in America, EnglewoodCliffs: Prentice-Hall Inc.

Landtag Thüringen Drucksache 5/8080 (16.07.2014): Bericht des Untersuchungsausschusses 5/1 „Rechtsterrorismus und Behördenhandeln“. Abrufbar unter: http://www.thueringerlandtag.de/imperia/md/content/landtag/aktuell/2014/drs58080.pdf (Stand: 10.10.2015).

Lea, John (2000): The Macpherson Report and the question of institutional racism. In: The Howard Journal, 39 (3), S. 219-233.

Lewicki, Aleksandra (2016 i.E.): Institutionelle Diskriminierung. Rechtliche Möglichkeiten in Deutschland und Großbritannien. In: Meral El und Karim Fereidooni (Hrsg.), Rassismuskritik und Widerstandsformen. National und international vergleichende Formen von Rassismus und Widerstand, Wiesbaden: Springer VS.

McLaughlin, Eugene/Murji, Karim (1999): After the Stephen Lawrence Report. In: Critical Social Policy, 19 (3), S: 371-385.

Miles, Robert (1992): Rassismus. Eine Einführung in die Geschichte und Theorie eines Begriffs. Zweite Auflage, Hamburg: Argument-Verlag.

Patel, Tina/Tyrer, David (2011): Race, crime and resistance, London: SAGE Publications Ltd.

Phillips, Coretta (2011): Institutional Racism and Ethnic Inequalities: An Expanded Multilevel Framework. In: Journal of Social Policy, 40(1), S. 173-192.

Rommelspacher, Birgit (2009): Was ist eigentlich Rassismus, In: Claus Melter und Paul Mecheril (Hrsg): Rassismuskritik, Rassismustheorie und -forschung, Schwalbach: Wochenschau.

Şimşek, Semiya/Schwarz, Peter (2013): Schmerzliche Heimat. Deutschland und der Mord an meinem Vater, Berlin: Rowohlt.

Singh, Gurchand (2000): The Concept and Context of Institutional Racism, In: Alan Marlow und Barry Loveday (Hrsg.): After Macpherson. Policing after the Stephen Lawrence Inquiry, Lyme Regis: Russel House Publishing, S. 29-40.

Solomos, John (1999): Social research and the Stephen Lawrence Inquiry. In: Sociological Research Online, 4 (1)

Terkessidis, Marc (1998): Psychologie des Rassismus, Wiesbaden: Westdeutscher Verlag.

Terkessidis, Marc (2004): Die Banalität des Rassismus, Bielefeld: transcript Verlag.

Türkische Gemeinde in Deutschland (2013): Bericht der Türkischen Gemeinde in Deutschland zum NSU und die daraus folgenden Konsequenzen. Abrufbar unter: http://tgd.de/wpcontent/uploads/2013/09/2013.08.27 TGD NSU-Bericht.pdf (Stand: 10.10.2015).

von der Behrens, Antonia/Daimagüler, Mehmet/Elberling, Björn/Fresenius, Berthold/Hoffmann, Alexander/Ilius, Carsten/Kolloge, Detlef/Kuhn, Stephan/Lex, Angelika/Lucas, Stephan /Parlayan, Ogün/Rabe, Jens/Reinecke, Eberhard/Sariyar, Aziz/Scharner, Sebastian/Schön, Reinhard/Stolle, Peer (22.08.2013): Der Fehler liegt im System, Pressemitteilung. In: Mut gegen rechte Gewalt. Abrufbar unter: http://www.mut-gegen-rechte-gewalt.de/news/meldung/derfehler-liegt-im-system-2013-08 (Stand: 10.10.2015).

Williams, Jenny (1985): Redefining Institutional Racism. In: Ethnic and Racial Studies, 8 (3), S. $323-$ 348. 